\title{
BMJ Open The efficacy and safety of complete pericardial drainage by means of intrapericardial fibrinolysis for the prevention of complications of pericardial effusion: a systematic review protocol
}

\author{
Aloysious Kakia, ${ }^{1}$ Charles S Wiysonge, ${ }^{2,3}$ Eleanor A Ochodo, ${ }^{2}$ \\ Abolade A Awotedu, ${ }^{4}$ Arsen D Ristic, ${ }^{5}$ Bongani M Mayosi ${ }^{6}$
}

To cite: Kakia $A$,

Wiysonge CS, Ochodo EA, et al. The efficacy and safety of complete pericardial drainage by means of intrapericardial fibrinolysis for the prevention of complications of pericardial effusion: a systematic review protocol. BMJ Open 2016;6:e007842.

doi:10.1136/bmjopen-2015007842

- Prepublication history for this paper is available online. To view these files please visit the journal online (http://dx.doi.org/10.1136/ bmjopen-2015-007842).

Received 8 February 2015 Revised 28 September 2015 Accepted 30 September 2015

\section{CrossMark}

For numbered affiliations see end of article.

Correspondence to Professor Charles S Wiysonge;

charlesw@sun.ac.za

\section{ABSTRACT}

Introduction: Intrapericardial fibrinolysis has been proposed as a means of preventing complications of pericardial effusion such as cardiac tamponade, persistent and recurrent pericardial effusion, and pericardial constriction. There is a need to understand the efficacy and safety of this procedure because it shows promise.

Methods and analysis: We aim to assess the effects of intrapericardial fibrinolysis in the treatment of pericardial effusion. We will search PubMed, the Cochrane Library, African Journals online, Cumulative Index to Nursing and Allied Health Literature, Trip database, Clinical trials.gov and the WHO International Clinical Trials Registry Platform for studies that evaluate the efficacy and/or safety of complete pericardial fluid drainage by intrapericardial fibrinolysis irrespective of study design, geographical location, language, age of participants, aetiology of pericarditis or types of fibrinolytics. Two authors will do the search independently, screen the search outputs for potentially eligible studies and assess whether the studies meet the inclusion criteria. Discrepancies between the two authors will be resolved through discussion and arbitration by a third author. Data from the selected studies shall be extracted using a standardised data collection form which will be piloted before use. The methodological quality of studies will be assessed using the Cochrane Collaboration's tools for assessing risk of bias for experimental studies and nonrandomised studies, respectively. The primary metaanalysis will use random effects models due to expected interstudy heterogeneity. Dichotomous data will be analysed using relative risk and continuous with data mean differences, both with $95 \% \mathrm{Cls}$.

Ethics and dissemination: Approval by an ethics committee is not required for this study as it is a protocol for a systematic review of published studies. The results will be disseminated through a conference presentation and peer-reviewed publication.

\section{Strengths and limitations of this study}

- The planned review will shed light on the evidence to date regarding the efficacy and safety of intrapericardial fibrinolysis in preventing complications of pericardial effusion, and guide future research on this theme.

- This manuscript is prepared according to the recent Preferred Reporting Items for Systematic review and Meta-Analysis Protocols (PRISMA-P) Statement.

- Unbiased selection of many studies conducted in different settings will strengthen the validity of the review results.

- The main limitation of the planned review will be the heterogeneity of the settings and designs of included studies.

Review registration number: PROSPERO, CRD42014015238.

\section{BACKGROUND}

The era of HIV has seen an increase in the incidence of pericarditis. ${ }^{1}$ The main cause of pericarditis in Africa is tuberculosis. ${ }^{2}$ Pericarditis may complicate to tamponade in the short term, and chronic effusive pericarditis and constrictive pericarditis in the long term. Cardiac tamponade and constrictive pericarditis lead to death if not treated in time. The definitive management of constrictive pericarditis involves pericardiectomy, which is associated with a mortality of up to $14 \%^{3}$ and is an expensive procedure. ${ }^{4}$ Imazio et $a \tilde{\bar{l}}$ have shown that tuberculous and purulent pericarditis are more likely to progress 
to constrictive pericarditis than pericarditis due to other causes. Ntsekhe et $a t^{6}$ found a $10.9 \%$ incidence of constrictive pericarditis over a 6 -month period in patients with pericardial effusions that were presumed to be tuberculous. These findings highlight the importance of efforts to prevent progression of pericarditis to constrictive pericarditis.

Various strategies have been used to prevent progression of acute pericarditis to constrictive pericarditis. Early diagnosis and prompt treatment of pericarditis, including treating the underlying cause and draining of effusions, are a major step in this direction. The use of colchicine as adjunctive treatment to prevent recurrent and persistent pericarditis, and thereby reducing the risk of constriction, showed promise in a randomised clinical trial conducted by Imazio et al. ${ }^{7}$ Corticosteroids have been found to be useful in several trials; however, the findings of Mayosi $e t a l^{8}$ have shown that corticosteroids could increase the risk of cancers in patients coinfected with HIV.

Intrapericardial fibrinolysis has been proposed as a way of stemming the development of cardiac tamponade and constriction in patients with effusive pericarditis. The objective of fibrinolysis is to target fibrin formation, to optimise evacuation of a thick fluid, and therefore to prevent both persistent purulent pericarditis and constrictive pericarditis. ${ }^{9}$ The procedure is also minimally invasive. A clinical review conducted by Augustin et a 9 concluded that intrapericardial fibrinolysis may be useful for prevention of constrictive pericarditis. Cui et $a l^{10}$ investigated the efficacy of intrapericardial fibrinolysis in preventing constrictive pericarditis in patients with infective pericardial effusion, $60 \%$ of which were of tuberculous origin. They found that the early employment of fibrinolysis optimised complete evacuation of the pericardial effusion, significantly reduced progress to pericardial constriction and was safe.

In view of the promise held by intrapericardial fibrinolysis, there is currently a need to better understand the safety and efficacy of the procedure. We propose, therefore, to conduct a systematic review to assess the efficacy and safety of intrapericardial fibrinolysis in the prevention of complications of pericardial effusion such as cardiac tamponade, recurrent or persistent effusion, constrictive pericarditis, hospitalisation and death.

\section{OBJECTIVES}

1. To determine whether complete pericardial drainage by intrapericardial fibrinolysis reduces the incidence of cardiac tamponade, persistent or recurrent pericardial effusion, constrictive pericarditis, hospitalisation and death in patients with pericardial effusion.

2. To determine whether complete pericardial drainage by intrapericardial fibrinolysis can be performed safely with respect to the incidence of haemorrhage, procedure-related cardiac tamponade, allergy and serious and non-serious adverse events.
3. To determine the appropriate timing, dose and volume of intrapericardial fibrinolysis.

\section{METHODS}

\section{Types of studies}

We will consider primary studies with the following designs:

- Intervention studies: randomised controlled trials (RCTs), and quasi-RCTs.

- Observational studies: case reports, cohort studies, case-control studies and cross-sectional studies.

\section{Types of participants}

People of all ages requiring intrapericardial fibrinolysis for evacuation of pericardial effusion due to any cause.

\section{Study settings}

We will include studies that evaluate the efficacy and/or safety of intrapericardial fibrinolysis for preventing constrictive pericarditis irrespective of geographical location.

\section{Types of interventions}

All types of fibrinolytics will be considered including (but not limited to) urokinase, streptokinase and tissue plasminogen activator.

\section{Types of outcome measures}

The efficacy outcomes of interest to this review are cardiac tamponade, persistent or recurrent pericardial effusion, constrictive pericarditis, hospitalisation and death in patients with pericardial effusion.

The safety outcomes will be the incidence of haemorrhage, procedure-related cardiac tamponade, allergy, serious and non-serious adverse events.

\section{Search methods for identification of studies}

We will develop a comprehensive strategy to search for all eligible studies available up to the search date, regardless of language or publication status. For published literature we will search the electronic databases PubMed, Cochrane Library (Cochrane Central Register of Controlled Trials, Cochrane Database of Systematic Reviews, Database of Abstracts of Reviews of Effects (DARE)), African Journals online (AJOL), Cumulative Index to Nursing and Allied Health Literature (CINAHL) and Trip database. We will use a combination of the following search terms and tailor them appropriately to the different databases: 'Pericarditis', 'tuberculous pericarditis,' 'purulent pericarditis,' 'pericardiocentesis', 'therapeutic pericardiocentesis,' 'fibrinolytics,' 'intrapericardial fibrinolytics,' 'urokinase,' 'streptokinase' and 'tissue plasminogen activator'. Box 1 below gives the provisional search strategy for PubMed, which will be adapted for each electronic database. To avoid selection bias, two authors will do the search independently. To access unpublished literature, we will 


\section{Box 1 Provisional search strategy for PubMed}

(((((((pericardiocentesis) OR 'pericardial drainage)' OR 'intrapericardial fibrinolysis)' OR fibrinolysis)) OR (((('tissue plasminogen activator)' OR urokinase) OR streptokinase) OR fibrinolytics))) AND $(((()((()(($ pericarditis $) \quad O R$ 'tuberculous pericarditis)' OR 'pericardial effusion)' OR 'TB pericarditis)' OR 'purulent pericarditis)' OR 'complicated pericarditis)' OR 'complications of pericarditis)' $\mathrm{OR}$ 'complications of pericardial effusions)' $\mathrm{OR}$ 'constrictive pericarditis)' $\mathrm{OR}$ 'recurrent pericardial effusions)' $\mathrm{OR}$ 'persistent pericarditis)' OR 'cardiac tamponade)'.

contact experts in the field of therapeutic pericardiocentesis and search Clinical trials.gov, and the WHO International Clinical Trials Registry Platform.

\section{Data collection and analysis}

Two authors will independently screen the search outputs for potentially eligible studies, compare their results and resolve disagreements by discussion and consensus. The two authors will then independently go through the full text of all potentially eligible studies to assess whether the studies meet the inclusion criteria defined by the study design, setting, intervention and outcomes. Discrepancies in the list of eligible studies between the two authors will be resolved through discussion and consensus. A structured and standardised data collection form shall be developed for extracting data from the selected studies. The form will capture key study characteristics, including study design, participants, methods used for diagnosis of pericardial effusion (eg, echocardiography), aetiology of effusions, interventions, risk of bias and outcomes. Prior to use, the extraction form will be piloted on at least three studies identified randomly from the list of included studies.

The methodological quality of studies will be assessed using the Cochrane Collaboration's tool for assessing risk of bias for experimental studies ${ }^{11}$ and the 'Cochrane risk of bias assessment tool for nonrandomised studies of interventions' for other study designs. ${ }^{12}$

All eligible studies will be summarised and analysed using the Cochrane Review Manager software. ${ }^{11}$ Two authors will extract the data, one author will enter the data and the second author will recheck the entries. In the event of discrepancy, the authors shall discuss and resolve the disagreement by discussion and consensus, and if this fails to resolve the disagreement a third author will arbitrate. If the studies are sufficiently similar, we will combine the data using the random effects model. We will examine statistical heterogeneity between study results using the $\chi^{2}$ test of homogeneity (with a significance $\alpha$-level of 0.1 ). We shall quantify statistical heterogeneity between study results using the inconsistency index $\left(\mathrm{I}^{2}\right) .{ }^{13}{ }^{14}$ When studies cannot be combined for meta-analysis due to diversity of interventions, narrative syntheses will be conducted.
We will stratify analysis by aetiology of pericardial effusion (eg, tuberculous, bacterial), type of pericarditis (eg, effusive, effusive constrictive), modality for diagnosis of pericardial effusions and constriction (eg, use of echography, echography not used) and study design (eg, controlled trials, observational studies). For any meta-analysis involving 10 or more studies, we will use funnel plots to assess the possibility of publication bias. In addition, we will apply the GRADE system to assess the strength of the evidence from the review. ${ }^{15}$

\section{Reporting of protocol and systematic review}

We plan to report the findings of the review as recommended in the Preferred Reporting Items for Systematic reviews and Meta-Analyses (PRISMA) guidelines. ${ }^{16}$ In addition, we prepared the review protocol according to the Preferred Reporting Items for Systematic review and Meta-Analysis Protocols (PRISMA-P) statement. ${ }^{17}$

\section{Ethics and dissemination}

The planned systematic review is registered with the International Prospective Register of Systematic Reviews (PROSPERO), registration number CRD42014015238. Systematic reviews draw on data available in the public domain, and do not need formal ethical review and approval. The findings of this systematic review will be disseminated through peer-reviewed journal publications and conference presentations. To our knowledge, no systematic review on intrapericardial fibrinolysis for the prevention of complications of pericardial effusion has been performed to date. Our discussion of the findings shall be in the light of the relevance of these data in clinical decision-making, and the future research design and direction on this topic.

\section{Author affiliations}

${ }^{1}$ Department of Family Medicine and Rural Health, Walter Sisulu University, Mthatha, South Africa

${ }^{2}$ Centre for Evidence-based Health Care, Stellenbosch University, Cape Town, South Africa

${ }^{3}$ Cochrane South Africa, South African Medical Research Council, Cape Town, South Africa

${ }^{4}$ Department of Medicine, Nelson Mandela Academic Hospital and Walter Sisulu University, Mthatha, South Africa

${ }^{5}$ Department of Cardiology, Clinical Center of Serbia and Belgrade University School of Medicine, Belgrade, Serbia

${ }^{6}$ Department of Medicine, Groote Schuur Hospital and University of Cape Town, Cape Town, South Africa

Twitter Follow Charles Wiysonge at @CharlesShey

Acknowledgements The authors acknowledge the Editor and peer reviewers for critical and constructive comments, which helped to improve earlier versions of the manuscript.

Contributors BMM conceived the study, and $A K$ and EAO wrote the first draft of the protocol. AK, CW, EO, AA, AR and BM critically revised successive drafts of the manuscript and approved the final version for publication. CW prepared the final version and is the guarantor of the manuscript.

Competing interests None declared.

Provenance and peer review Not commissioned; externally peer reviewed. 
Open Access This is an Open Access article distributed in accordance with the Creative Commons Attribution Non Commercial (CC BY-NC 4.0) license, which permits others to distribute, remix, adapt, build upon this work noncommercially, and license their derivative works on different terms, provided the original work is properly cited and the use is non-commercial. See: http:// creativecommons.org/licenses/by-nc/4.0/

\section{REFERENCES}

1. Mayosi BM, Burgess LJ, Doubell AF. Tuberculous pericarditis. Circulation 2005:112:3608-16.

2. Mayosi BM. Contemporary trends in the epidemiology and management of cardiomyopathy and pericarditis in sub-Saharan Africa. Heart 2007;93:1176-83.

3. Mutyaba A, Balkaran S, Cloete R, et al. Constrictive pericarditis requiring pericardiectomy at Groote Schuur Hospital in Cape Town, South Africa: causes and perioperative outcomes in the HIV era (1990-2012). J Thorac Cardiovasc Surg 2014;148:3058-65.e1.

4. Mayosi BM, Ntsekhe M, Volmink JA, et al. Interventions for treating tuberculous pericarditis. Cochrane Database Syst Rev 2002;(4): CD000526.

5. Imazio M, Brucato A, Maestroni S, et al. Risk of constrictive pericarditis after acute pericarditis. Circulation 2011;124:1270-5.

6. Ntsekhe M, Wiysonge CS, Gumedze F, et al. HIV infection is associated with a lower incidence of constriction in presumed tuberculous pericarditis: a prospective observational study. PLOS ONE 2008;3:e2253.

7. Imazio M, Brucato A, Cemin R, et al. Colchicine for recurrent pericarditis (CORP): a randomized trial. Ann Intern Med 2011:155:409-14.
8. Mayosi BM, Ntsekhe M, Bosch J, et al. Prednisolone and Mycobacterium indicus pranii in tuberculous pericarditis. N Engl $J$ Med 2014;371:1121-30.

9. Augustin P, Desmard M, Mordant P, et al. Clinical review: intrapericardial fibrinolysis in management of purulent pericarditis. Crit Care 2011;15:220.

10. Cui HB, Chen XY, Cui CC, et al. Prevention of pericardial constriction by transcatheter intrapericardial fibrinolysis with urokinase. Chin Med Sci J 2005;20:5-10.

11. Higgins JPT, Green S, eds. Cochrane handbook for systematic reviews of interventions version 5.1.0[updated March 2011]. Oxford, UK: The Cochrane Collaboration, 2011. Available from http:// handbook.cochrane.org/ (accessed 28 Dec 2015).

12. Sterne JAC, Higgins JPT, Reeves BC, on behalf of the development group for ACROBAT-NRSI. A Cochrane Risk Of Bias Assessment Tool: for Non-Randomized Studies of Interventions (ACROBATNRSI), Version 1.0.0, 24 September 2014. http://www.riskofbias.info (accessed 28 Sep 2015).

13. Hardy RJ, Thompson SG. Detecting and describing heterogeneity in meta-analysis. Stat Med 1998;17:841-56.

14. Higgins JP, Thompson SG, Deeks JJ, et al. Measuring inconsistency in meta-analysis. BMJ 2003;327:557-60.

15. Guyatt G, Oxman AD, Akl EA, et al. GRADE guidelines: 1. Introduction-GRADE evidence profiles and summary of findings tables. J Clin Epidemiol 2011;64:383-94.

16. Liberati A, Altman DG, Tetzlaff $J$, et al. The PRISMA statement for reporting systematic reviews and meta-analyses of studies that evaluate healthcare interventions: explanation and elaboration. $B M J$ 2009;339:b2700.

17. Shamseer L, Moher D, Clarke M, et al, PRISMA-P Group. Preferred reporting items for systematic review and meta-analysis protocols (PRISMA-P) 2015: elaboration and explanation. BMJ 2015;349:g7647. 